\title{
Socioeconomic inequalities in common mental disorders and psychotherapy treatment in the UK between 1991 and 2009'
}

\author{
Markus Jokela, G. David Batty, Jussi Vahtera, Marko Elovainio and Mika Kivimäki
}

\section{Background}

Inequality in health and treatment of disease across socioeconomic status groups is a major public health issue.

\section{Aims}

To examine differences in socioeconomic status in common mental disorders and use of psychotherapy provided by the public and private sector in the UK between 1991 and 2009.

\section{Method}

During these years, 28054 men and women responded to annual surveys by the nationally representative, populationbased British Household Panel Survey (on average 7

measurements per participant; 207545 person-observations) In each year, common mental disorders were assessed with the self-reported 12-item General Health Questionnaire and socioeconomic status was assessed on the basis of household income, occupational status and education.

\section{Results}

Higher socioeconomic status was associated with lower odds of common mental disorder (highest $v$. lowest household income quintile odds ratio (OR) $0.88,95 \% \mathrm{Cl} 0.82-0.94)$ and

of being treated by publicly provided psychotherapy

$(\mathrm{OR}=0.43,95 \% \mathrm{Cl}$ 0.34-0.55), but higher odds of being a client of private psychotherapy $(\mathrm{OR}=3.33,95 \% \mathrm{Cl} 2.36-4.71)$. The status difference in publicly provided psychotherapy treatment was more pronounced at the end of follow-up $(\mathrm{OR}=0.36,95 \% \mathrm{Cl} 0.23-0.56$, in 2005-2009) than at the beginning of the follow-up period $(\mathrm{OR}=0.96,95 \% \mathrm{Cl} 0.66-$ 1.39, in 1991-1994; time interaction $P<0.001$ ). The findings for occupational status and education were similar to those for household income.

\section{Conclusions}

The use of publicly provided psychotherapy has improved between 1991 and 2009 among those with low socioeconomic status, although social inequalities in common mental disorders remain.

\section{Declaration of interest}

None.
Psychotherapy is one of the recognised treatments for various mental health problems, ${ }^{1}$ particularly for depressive and anxiety disorders, which represent the major burden of mental illness in the general population. ${ }^{2,3}$ Although social inequalities in the incidence, prevalence and course of common mental disorders are well documented, ${ }^{4-8}$ the patterns of psychotherapy treatment associated with socioeconomic status are surprisingly underexplored. $^{9-11}$ In France and Finland, higher educational level has been shown to be associated with higher probability of having psychotherapy. ${ }^{9,12}$ In a British sample, people living in more deprived areas were more likely to consult a general practitioner for mental health problems compared with those living in affluent neighbourhoods, ${ }^{13}$ while another British study found no association between neighbourhood deprivation or affluence and referrals to, or acceptance of, psychotherapy. ${ }^{14}$ In the 1993 National Survey of Psychiatry Morbidity in Britain, social class was not related to receiving treatment for neurotic disorders. ${ }^{15}$ Given the sparseness of data to date, we examined socioeconomic status in relation to common mental disorder and psychotherapy treatment in the UK between 1991 and 2009.

\section{Method}

Our sample comprised participants in the British Household Panel Survey (BHPS), a longitudinal survey of a nationally representative sample of over 5000 British households with annual follow-up. ${ }^{16}$ The original cohort included 10264 individuals aged $16-97$ years $($ mean $=44.4$, s.d. $=18.3)$ at baseline in 1991, and was based on a clustered, stratified sample of

'See editorial, pp. 86-88, this issue. addresses throughout Great Britain south of the Caledonian Canal (i.e. excluding the north of Scotland and Northern Ireland). New participants were included in the sample over the years if they were born to an original sample member, if they had moved into a household in the original sample or if a member of the original sample moved into a new household with one or more new people. In addition, the sample was enriched with additional recruitment of participants at waves 9 and 11, from Scotland and Wales and from Northern Ireland respectively, so extending the sample to cover the whole of the UK. The most recent (18th) follow-up of the BHPS took place in 2008-2009, after which the cohort has become part of the larger Understanding Society Study (www.understandingsociety.org.uk/).

For the present analysis we included all person-observations of participants for whom information on measures of socioeconomic status, common mental disorder, psychotherapy treatment and covariates was available ( $n=28054$ unique individuals; 207545 person-observations). The sample size was slightly smaller in the analysis of common mental disorder and psychotherapy ( $n=22586 ; 174630$ person-observations), for which each personobservation required data from two consecutive study waves (common mental disorder at baseline and psychotherapy treatment reported in the next wave). To take into account non-response, all analyses were weighted using cross-sectional sample weights.

\section{Measures}

At each study wave common mental disorders were identified using the 12-item General Health Questionnaire (GHQ). ${ }^{3,17}$ The items were scored using the GHQ scoring method (total scale range 0-12) and GHQ caseness was defined as a score above 2. 
At each study wave the participants were requested to report whether they had used different health and welfare services ('Here is a list of some health and welfare services. Have you yourself made use of any of these services since September 1st last year?'). One of the items was 'Psychotherapist (including psychiatrist or analyst)' (no/yes), and the respondent was also requested to indicate whether this had been provided by the public (National Health Service, NHS) or the private sector.

Socioeconomic status was measured at each study wave by three indicators:

(a) annual household income from all income sources (categorised into quintiles at each year);

(b) educational level, stratified into four groups: 0, primary education or less; 1, secondary education (Certificate of Secondary Education, O-level); 2, tertiary education (A-level or diploma); 3 , higher education (higher or first degree);

(c) occupational social class of the participant's most recent job, classified according to the Registrar General's scheme: 0, unskilled/partly skilled/never had a job; 1, skilled manual; 2, skilled non-manual; 3 , managerial or professional.

Given the way the time interval for psychotherapy treatment was framed (see above), it had some variability across participants (average interval length 13.2 months, s.d.=1.6). We therefore included time interval as a covariate in models predicting psychotherapy treatment. In addition, all models were adjusted for age, birth year, gender, ethnicity ( 0 , White; 1 , other ethnicity, based on the participant's self-reported ethnic background), subsample ( 0 , original sample; 1 , Wales; 2 , Scotland; 3 , Northern Ireland), marital status (0, married; 1 , never married; 2 , separated/ divorced; 3 , widowed; 4, civil partnership) and number of children (top-coded to four or more children). Two of the latter covariates were included to take into account the fact that a given household income level has different meanings for, say, a person living alone and for a couple with four children.

\section{Statistical analysis}

Given the longitudinal study design with annual surveys, each participant could contribute between 1 and 18 person-observations to the data-set (on average 7 measurements per participant). Psychotherapy treatment, GHQ caseness, socioeconomic status, marital status, number of children and age were treated as timedependent variables, that is, the same participant could have different values in each person-observation (information for these covariates were reported at each wave). Gender, birth year, ethnicity and subsample membership were time-independent variables. The number of participants and person-observations included in each analysis varied somewhat depending on the availability of data for the specific socioeconomic status indicator.

To take into account the non-independence of the personobservations from the same individuals over time, we fitted logistic and multinomial logistic models using robust estimator with person as the clustering variable. This approach treats the repeated measures essentially as repeated cross-sectional data collections without considering longitudinal effects within individuals. Time trends in GHQ caseness and psychotherapy were assessed by predicting the outcomes with interaction effects between socioeconomic status and categorically coded year of interview (1991-1994, 1995-1999, 2000-2004, 2005-2009). When examining psychotherapy use according to GHQ caseness status, we used time-lagged GHQ status as the exposure, i.e. caseness in the previous study wave was used to predict psychotherapy use at the following study wave.

\section{Results}

Just over half of the 28054 participants were women (53.0\%) and $84.9 \%$ were White, with an average age of 45.2 years (s.d.=18.1) and birth year of 1955 (s.d. 19). The mean duration of follow-up for a participant was 11.2 years (s.d.=5.5). Table 1 shows the number of participants by study year and subsample group. Individuals and family members of the original sample composed $61.4 \%$ of the participants and $72.1 \%$ of the personobservations. Descriptive statistics of the study variables are reported in online Table DS1. Average prevalence of GHQ caseness and psychotherapy treatment per year was $26.0 \%$ and $2.1 \%$ respectively, with no marked change over the follow-up period. In total, $8.6 \%$ of the participants reported having had psychotherapy treatment at least once during the follow-up period. Of all the reported psychotherapy treatments (4274 person-observations from 2410 different individuals), $77.5 \%$ were provided by public health services, $21.3 \%$ by the private sector and $1.2 \%$ by both (the last group was excluded from the provider-specific analyses presented below).

\section{Association between GHQ caseness and psychotherapy}

Common mental disorders were more likely to precede publicly than privately provided psychotherapy treatment (Table 2), indicating lesser mental distress among clients of private compared with public psychotherapy. However, this difference attenuated over time $(P=0.01)$ because the association between GHQ caseness and privately provided psychotherapy strengthened over time (Table 2).

\section{Social inequalities in GHQ caseness and psychotherapy treatment}

A socioeconomic gradient in GHQ caseness was observed (Table 3), with higher household income and higher occupational status being associated with lower risk of GHQ caseness. High

\begin{tabular}{|c|c|c|c|c|c|}
\hline Wave & $\begin{array}{l}\text { Original } \\
\text { sample }\end{array}$ & Wales & Scotland & $\begin{array}{l}\text { Northern } \\
\text { Ireland }\end{array}$ & Total \\
\hline 1 & 9658 & & & & 9658 \\
\hline 2 & 9170 & & & & 9170 \\
\hline 3 & 8830 & & & & 8830 \\
\hline 4 & 8813 & & & & 8813 \\
\hline 5 & 8594 & & & & 8594 \\
\hline 6 & 8897 & & & & 8897 \\
\hline 7 & 8850 & & & & 8850 \\
\hline 8 & 8741 & & & & 8741 \\
\hline 9 & 8542 & 2240 & 2250 & & 13032 \\
\hline 10 & 8401 & 2179 & 2384 & & 12964 \\
\hline 11 & 8254 & 2174 & 2259 & 3029 & 15716 \\
\hline 12 & 8051 & 2053 & 2060 & 2558 & 14722 \\
\hline 13 & 7835 & 2003 & 2002 & 2473 & 14313 \\
\hline 14 & 7643 & 1928 & 1886 & 2228 & 13685 \\
\hline 15 & 7563 & 1941 & 1865 & 2230 & 13599 \\
\hline 16 & 7499 & 1949 & 1776 & 1942 & 13166 \\
\hline 17 & 7328 & 1872 & 1700 & 1843 & 12743 \\
\hline 18 & 7022 & 1812 & 1648 & 1570 & 12052 \\
\hline Total & 149691 & 20151 & 19830 & 17873 & 207545 \\
\hline
\end{tabular}


Table 2 Caseness defined by General Health Questionnaire score as a predictor of subsequent publicly or privately provided psychotherapy treatment

\begin{tabular}{|c|c|c|c|c|}
\hline \multirow[b]{2}{*}{ Period } & \multicolumn{2}{|c|}{ OR $(95 \% \mathrm{Cl})^{\mathrm{a}}$} & \multicolumn{2}{|c|}{ GHQ cases, ${ }^{\mathrm{b}} \%$} \\
\hline & Public & Private & Public & Private \\
\hline 1991-1994 & $3.81(2.97-4.89)$ & 1.79 (1.09-2.96) & 57 & 38 \\
\hline 1995-1999 & $4.20(3.44-5.12)$ & 1.59 (1.08-2.32) & 60 & 35 \\
\hline 2000-2004 & $4.63(3.78-5.68)$ & $2.53(1.81-3.53)$ & 61 & 45 \\
\hline 2005-2009 & $4.49(3.53-5.71)$ & $3.19(2.15-4.74)$ & 60 & 52 \\
\hline Total & 4.39 (3.86-4.98) & $2.29(1.87-2.80)$ & 60 & 43 \\
\hline
\end{tabular}

educational level was also associated with lower GHQ caseness risk, except for the highest educational group. The association between socioeconomic status and use of psychotherapy was dependent on the provider: high status was associated with lower odds of having publicly provided psychotherapy but higher odds of having privately provided psychotherapy (Table 3). Although the associations were substantial for both forms of psychotherapy, socioeconomic status was more strongly associated with private than public psychotherapy, education being the most prominent factor.

There was no evidence of change in social inequalities in GHQ caseness (Fig. 1; $P>0.18$ for all interactions between time and socioeconomic status indicators) or in use of private psychotherapy during the years 1991-2009 ( $P>0.30$ for time interaction). In contrast, the socioeconomic gradient in public psychotherapy treatments widened over time (Fig. 2). The odds ratios for highest $v$. lowest income quintile were 0.96 (95\% CI $0.66-1.39)$ in $1991-1994$ and 0.36 (95\% CI $0.23-0.56)$ in $2005-$ 2009 (for 1 unit increase $\mathrm{OR}=0.97,95 \%$ CI $0.89-1.06$, and $\mathrm{OR}=0.78,95 \%$ CI $0.71-0.87$ respectively; $P<0.001$ for time interaction). The odds ratios for highest $v$. lowest occupational status were 0.86 (95\% CI $0.63-1.19)$ in 1991-1994 and 0.52 (95\% CI 0.37-0.74) in 2005-2009 (for 1 unit increase $\mathrm{OR}=0.94,95 \%$ CI $0.85-1.05$, and $\mathrm{OR}=0.82,95 \%$ CI $0.73-0.92$, respectively; $P=0.02$ for time interaction). A similar, although statistically non-significant, pattern of widening socioeconomic gradient in public psychotherapy treatments was observed when education was used as the indicator of socioeconomic status (for 1 unit increase OR $=1.03,95 \%$ CI $0.89-1.18$, in 1991-1994, and $\mathrm{OR}=0.86,95 \%$ CI $0.75-1.00$ in $2005-2009 ; P=0.21$ for time interaction).

\section{Sensitivity analyses}

To test whether the addition of the subsamples in waves 9 and 11 accounted for any of the observed associations or interaction effects with time, we refitted all the models using only data from the participants and family members of the original sample ( $n=17236 ; 149691$ person-observations). The results remained substantially the same as in the main analyses (data not shown).

\begin{tabular}{|c|c|c|c|}
\hline & \multicolumn{3}{|c|}{ Odds ratios $(95 \% \mathrm{Cl})^{\mathrm{a}}$} \\
\hline & \multirow[b]{2}{*}{ GHQ caseness } & \multicolumn{2}{|c|}{ Psychotherapy } \\
\hline & & Public & Private \\
\hline \multicolumn{4}{|l|}{ Household income } \\
\hline I (lowest 20\%) & 1.00 (reference) & 1.00 (reference) & 1.00 (reference) \\
\hline$\|$ & $1.10(1.03-1.17)$ & $1.12(0.94-1.33)$ & $0.96(0.64-1.45)$ \\
\hline III & $1.00(0.94-1.06)$ & $1.07(0.89-1.29)$ & $1.01(0.70-1.45)$ \\
\hline IV & $0.90(0.85-0.96)$ & $0.71(0.57-0.88)$ & $1.62(1.14-2.29)$ \\
\hline V (highest 20\%) & $0.88(0.82-0.94)$ & $0.43(0.34-0.55)$ & $3.33(2.36-4.71)$ \\
\hline Linear trend & $0.96(0.94-0.97)$ & $0.83(0.79-0.88)$ & $1.40(1.26-1.56)$ \\
\hline \multicolumn{4}{|l|}{ Education } \\
\hline Primary or less & 1.00 (reference) & 1.00 (reference) & 1.00 (reference) \\
\hline Secondary & $0.81(0.75-0.87)$ & $0.79(0.63-0.98)$ & $1.65(1.08-2.52)$ \\
\hline Tertiary & 0.87 (0.80-0.94) & $0.88(0.70-1.11)$ & $3.08(1.98-4.78)$ \\
\hline Higher education & $0.94(0.86-1.03)$ & $0.67(0.50-0.88)$ & $6.51(4.01-10.57)$ \\
\hline Linear trend & $0.98(0.95-1.01)$ & $0.90(0.83-0.98)$ & $1.90(1.64-2.21)$ \\
\hline \multicolumn{4}{|l|}{ Occupational status } \\
\hline Unskilled/partly skilled & 1.00 (reference) & 1.00 (reference) & 1.00 (reference) \\
\hline Skilled manual & $0.95(0.88-1.02)$ & $0.78(0.62-0.97)$ & $0.67(0.38-1.19)$ \\
\hline Skilled non-manual & $0.92(0.86-0.98)$ & $0.78(0.64-0.96)$ & $1.43(0.96-2.14)$ \\
\hline Managerial/professional & $0.92(0.87-0.98)$ & $0.61(0.50-0.74)$ & $2.73(1.78-4.17)$ \\
\hline Linear trend & $0.97(0.95-0.99)$ & $0.86(0.81-0.92)$ & $1.52(1.31-1.76)$ \\
\hline
\end{tabular}


(a)

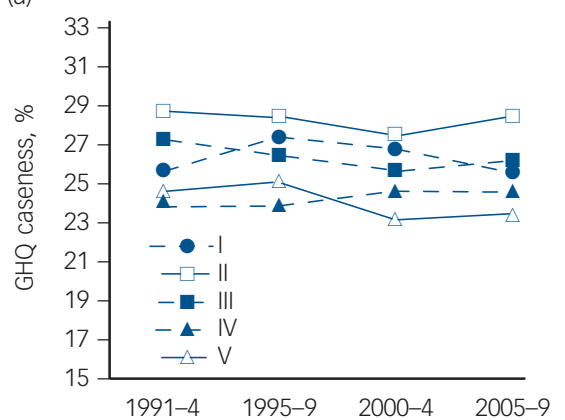

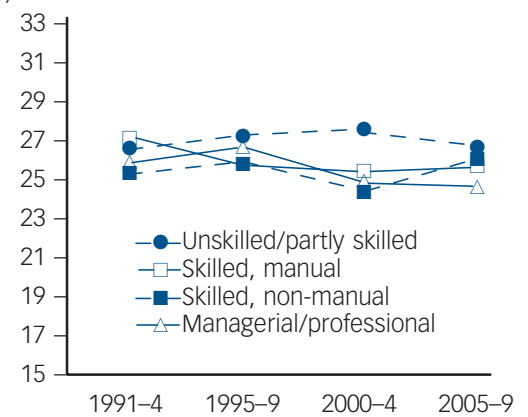

(c)

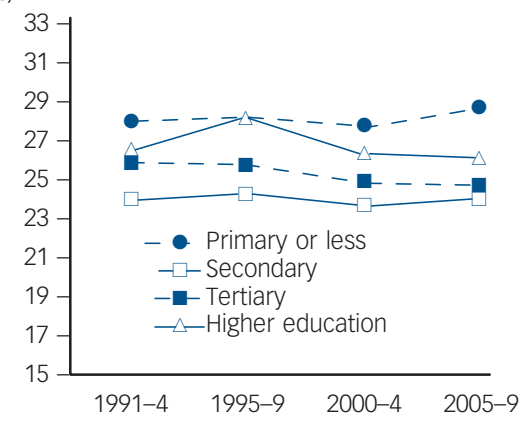

Fig. 1 Estimated prevalence of General Health Questionnaire (GHQ) cases categorised by socioeconomic status indicator and year: (a) income; (b) occupational class (I, lowest $20 \%$, V, highest $20 \%$ ); (c) education.

(a)

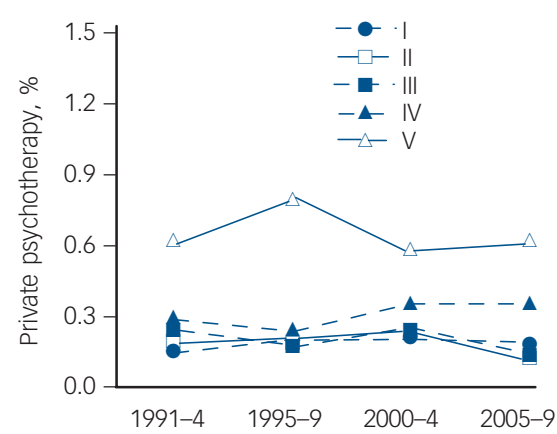

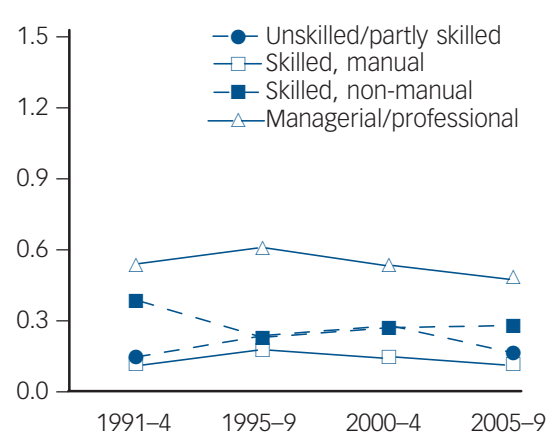

(c)

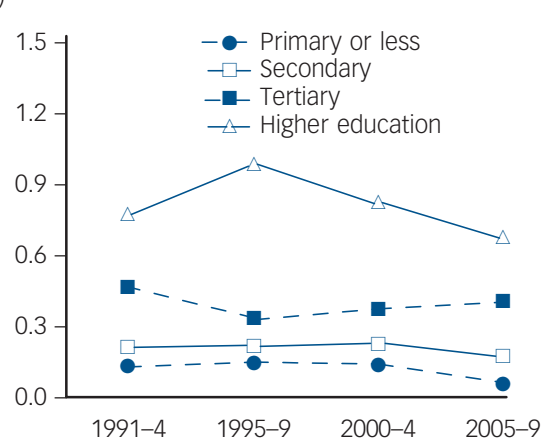

(d)

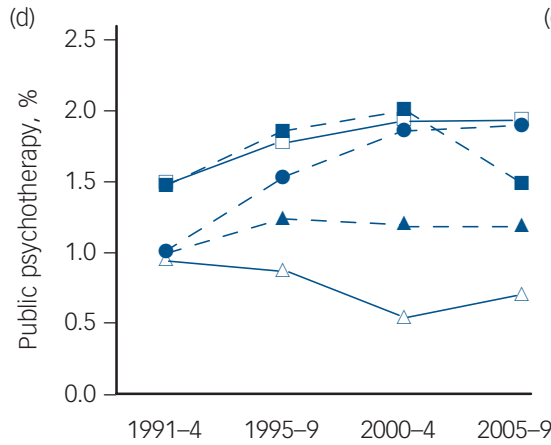

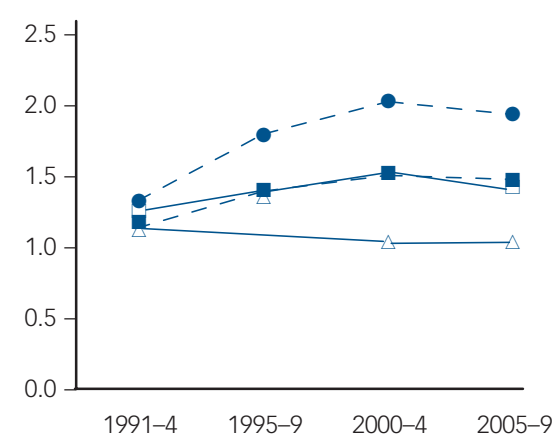

(f)

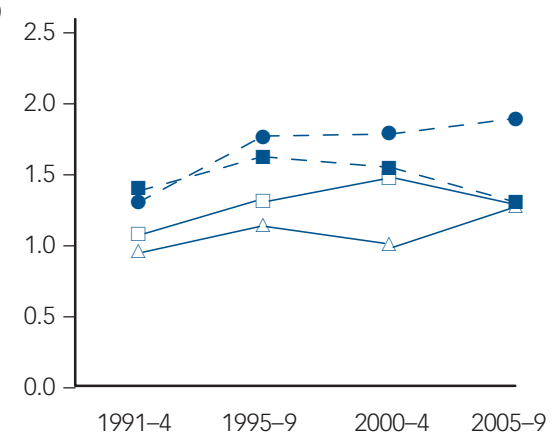

Fig. 2 Estimated prevalence of participants receiving privately and publicly provided psychotherapy treatment categorised by socioeconomic indicators and year. Privately provided: (a) income, (b) occupational class, (c) education. Publicly provided: (d) income, (e) occupational class, $(f)$ education.

The results were also essentially unchanged when the models were fitted without sample weights (data not shown). The associations between socioeconomic status and psychotherapy treatment were similar in GHQ cases and GHQ non-cases (assessed in the previous study wave), suggesting that the status differences in the use of psychotherapy were not specific to those with symptoms of psychological distress (online Table DS2).

\section{Discussion}

In a cohort of 28000 adults living in the UK between 1991 and 2009, we demonstrated socioeconomic inequalities in the prevalence of common mental disorders as indicated by the 12-item GHQ. Low education, household income and occupational status were also associated with greater odds of having psychotherapy treatment provided by the NHS. The socioeconomic gradients associated with publicly provided psychotherapy treatment widened between 1991-1994 and 2005-2009, suggesting that the treatment needs of those with the highest risk of common mental disorders were better met in the latter 5-year period than at the beginning of the 1990s. In contrast, the socioeconomic gradients associated with privately provided psychotherapy were inverse, its use being most common among those with high status (high education in particular), with no change over time.

Inequalities in health and treatment of disease between groups of different socioeconomic status are a major public health issue globally. According to the inverse care hypothesis, ${ }^{18}$ there is a mismatch between the distribution of care and clinical need, such that treatment rates tend to be lower in the disadvantaged groups despite their higher disease rates. In our study this hypothesis was 
supported in relation to socioeconomic status and use of privately provided psychotherapy treatment, which normally costs $£ 40-100$ per session. These findings accord with the large body of literature suggesting poorer care for mental health problems among people with low socioeconomic position. ${ }^{6,19-22}$ Higher socioeconomic status and education have been shown to correlate with greater probabilities of visiting a psychotherapist, ${ }^{9,12}$ and of taking antidepressant medication, ${ }^{6}$ suggesting that more wealthy and educated individuals are more likely to seek treatment even though they tend to experience a lower incidence of mental disorders compared with the socially disadvantaged.

Our findings on psychotherapy provided by the public sector did not support the inverse care hypothesis. Indeed, there appeared to be a favourable development over time indicating that publicly provided psychotherapeutic treatment is increasingly concentrated in the socioeconomic groups with the highest need, i.e. among those with low socioeconomic status. In a previous UK study, people living in more deprived areas were more likely to consult a general practitioner for mental health problems than those living in affluent areas, ${ }^{13}$ suggesting a similar socioeconomic pattern. Publicly provided psychotherapy is not as readily available as private psychotherapy. People seeking psychotherapy from the NHS first need a referral from their general practitioner (or other healthcare professional), and usually people need to wait for several months before receiving treatment.

The persistent socioeconomic gradient in common mental disorders needs to be considered in a broader context of health inequalities, ${ }^{23,24}$ because mental disorders increase the risk of various physical diseases, including chronic physical illness. ${ }^{2}$ Improving the balance between distribution of care and clinical need in relation to publicly provided mental health treatment might therefore contribute to overall reduction in socioeconomic inequalities in health. However, a corresponding favourable trend in the treatment of chronic physical illnesses is also needed in order to narrow social inequalities in mental disorders, because chronic physical diseases increase symptoms of common mental disorders. ${ }^{25}$

\section{GHQ caseness as proxy for mental distress}

Although GHQ caseness is not a precise indicator of the need for psychotherapy, it was strongly associated with subsequent psychotherapy use during the next year. Caseness can therefore be interpreted as a crude proxy for mental distress that might prompt individuals to seek psychotherapy. Clients of public and private psychotherapy differed in the frequency of common mental disorder preceding treatment: $60 \%$ of clients of publicly provided psychotherapy met criteria for GHQ caseness in the previous year compared with $43 \%$ of clients of privately provided psychotherapy. This implies that the psychological profiles of public $v$. private sector clients may exhibit important differences in terms of mental distress leading them to seek help. However, there was evidence for increasing rates of GHQ caseness among clients of privately provided psychotherapy from 1991-1994 to 2005-2009, suggesting that people seeking private psychotherapy today may be more mentally distressed than their counterparts in the early 1990s.

\section{Study limitations}

This study has several limitations. First, we measured common mental disorders with the 12-item GHQ which, although a validated screening measure, was not designed to make a psychiatric diagnosis. Our analysis thus cannot address directly the issue of unmet needs in mental health treatment. Second, information concerning psychotherapy treatment was based on a self-reported answer to a single question about use of the services of a psychotherapist (psychiatrist or analyst). This leaves out information on the indication, type, frequency, length or effectiveness of different psychotherapies. The use of, for example, cognitive-behavioural and psychoanalytically oriented therapies may follow different socioeconomic gradients and time trends, but our data were not capable of taking such differences into account. Third, we cannot exclude the possibility that socially patterned reporting biases may have affected our findings, e.g. it might have become more acceptable for individuals of low socioeconomic status to report having had psychotherapy. The strengths of the study, on the other hand, include a large, nationally representative sample, a long follow-up period and data on the use of public $v$. private psychotherapy.

\section{Study implications}

In summary, the recent socioeconomic patterns in use of publicly provided psychotherapy appear to present fairly positive trends in the UK. Low socioeconomic status was associated with higher likelihood of having publicly provided psychotherapy, which indicates an improving match between need and care for mental health treatment in socioeconomically disadvantaged groups. In contrast, private psychotherapy continues to be concentrated among those with high socioeconomic status. The existing socioeconomic gradients in common mental disorders demonstrate the continued need to combat socioeconomic inequalities in mental health in the UK.

\footnotetext{
Markus Jokela, PhD, Department of Epidemiology and Public Health, University College London, UK, Department of Psychology, Institute of Behavioural Sciences, University of Helsinki, and Finnish Institute of Occupational Health, Helsinki, Finland; G. David Batty, PhD, Department of Epidemiology and Public Health, University G. David Batty, PhD, Department of Epidemiology and Public Health, University
College London, and Centre for Cognitive Ageing and Cognitive Epidemiology, Department of Psychology, University of Edinburgh, UK; Jussi Vahtera, MD, Finnish Institute of Occupational Health, Helsinki, and Department of Public Health, University of Turku and Turku University Hospital, Turku, Finland; Marko Elovainio, PhD, National Institute for Health and Welfare, Helsinki, Finland; Mika Kivimäki, Department of Epidemiology and Public Health, University College London, UK

Correspondence: Markus Jokela, Department of Psychology, Institute of Behavioural Sciences, Siltavuorenpenger 1A, PO Box 9, 00014 University of Helsinki, Finland. Email: markus.jokela@helsinki.fi

First received 1 Jul 2011, final revision 12 Dec 2011, accepted 17 Jan 2012
}

\section{Funding}

The study was supported by the Academy of Finland (grant numbers 124322, 124271 and 132944) and the BUPA Foundation, UK, and Medical Research Council (MRC; grant G0902037). The Centre for Cognitive Ageing and Cognitive Epidemiology is supported by the Biotechnology and Biological sciences Research Council, the Engineering and Physical Sciences Research Council, the Economic and Social Research Council, the MRC and the University of Edinburgh as part of the cross-council Lifelong Health and Wellbeing initiative. G.D.B. is a Wellcome Trust Fellow.

\section{References}

1 Da Silva PFR, Blay SL. Prevalence and characteristics of outpatient psychotherapy use: a systematic review. J Nerv Ment Dis 2010; 198: 783-9.

2 Prince M, Patel V, Saxena S, Maj M, Maselko J, Phillips MR, et al. Global mental health - no health without mental health. Lancet 2007; 370: 859-77.

3 Goldberg DP. Detecting Psychiatric IIIness by Questionnaire. Oxford University Press, 1972.

4 Lorant V, Deliege D, Eaton W, Robert A, Philippot P, Ansseau M. Socioeconomic inequalities in depression: a meta-analysis. Am J Epidemiol 2003; 157: 98-112.

5 Stansfeld SA, Marmot MG. Social class and minor psychiatric disorder in British civil servants: a validated screening survey using the General Health Questionnaire. Psychol Med 1992; 22: 739-49. 
6 Kivimaki M, Gunnell D, Lawlor DA, Smith GD, Pentti J, Virtanen M, et al. Social inequalities in antidepressant treatment and mortality: a longitudinal register study. Psychol Med 2007; 37: 373-82.

7 Jokela M, Keltikangas-Jarvinen L. The association between low socioeconomic status and depressive symptoms depends on temperament and personality traits. Pers Individ Diff 2011; 51: 302-8

8 Jokela M, Singh-Manoux A, Ferrie JE, Gimeno D, Akbaraly TN, Shipley MJ, et al. The association of cognitive performance with mental health and physical functioning strengthens with age: the Whitehall II cohort study. Psychol Med 2010; 40: 837-45.

9 Briffault X, Sapinho D, Villamaux M, Kovess V. Factors associated with use of psychotherapy. Soc Psychiatry Psychiatr Epidemiol 2008; 43: 165-71.

10 Olfson M, Marcus SC, Druss B, Pincus HA. National trends in the use of outpatient psychotherapy. Am J Psychiatry 2002; 159: 1914-20.

11 Clarkin JF, Levy KN. The influence of client variables on psychotherapy. In Bergin \& Garfield's Handbook of Psychotherapy and Behavior Change (ed MJ Lambert): 194-226. Wiley, 2004.

12 Suoyrjö H, Hinkka K, Kivimäki M, Klaukka T, Pentti J, Vahtera J. Allocation of rehabilitation measures provided by the Social Insurance Institution in Finland: a register linkage study. J Rehabil Med 2007; 39: 198-204.

13 Oliver Ml, Pearson N, Coe N, Gunnell D. Help-seeking behaviour in men and women with common mental health problems: cross-sectional study. Br J Psychiatry 2005; 186: 297-301.

14 Saxon D, Fitzgerald G, Houghton S, Lemme F, Saul C, Warden S, et al. Psychotherapy provision, socioeconomic deprivation, and the inverse care law. Psychother Res 2007; 17: 515-21.

15 Bebbington PE, Brugha TS, Meltzer $\mathrm{H}$, Jenkins $\mathrm{R}$, Ceresa $\mathrm{C}$, Farrell $\mathrm{M}$, et al. Neurotic disorders and the receipt of psychiatric treatment. Psychol Med 2000; 30: 1369-76.
16 Taylor MF, Brice J, Buck N, Prentice-Lane E. British Household Panel Survey User Manual. Vol A: Introduction, Technical Report and Appendices. University of Essex, 2010.

17 Pevalin DJ. Multiple applications of the GHQ-12 in a general population sample: an investigation of long-term retest effects. Soc Psychiatry Psychiatr Epidemiol 2000; 35: 508-12.

18 Hart JT. Inverse care law. Lancet 1971; 1: 405-12.

19 Howard KI, Cornille TA, Lyons JS, Vessey JT, Lueger RJ, Saunders SM. Patterns of mental health service utilization. Arch Gen Psychiatry 1996; 53: 696-703.

20 Muntaner C, Wolyniec P, McGrath J, Pulver AE. Differences in social class among psychotic patients at patient admission. Psychiatr Serv 1995; 46: $176-8$.

21 Muntaner C, Wolyniec P, McGrath J, Pulver AE. Psychotic inpatients' socia class and their first admission to state or private psychiatric Baltimore hospitals. Am J Public Health 1994; 84: 287-9.

22 Muntaner C, Eaton WW, Miech R, O'Campo P. Socioeconomic position and major mental disorders. Epidemiol Rev 2004; 26: 53-62.

23 Weich $\mathrm{S}$, Lewis $\mathrm{G}$, Jenkins SP. Income inequality and the prevalence of common mental disorders in Britain. Br J Psychiatry 2001; 178: 222-7.

24 Wilkinson RG, Pickett KE. Income inequality and population health: a review and explanation of the evidence. Soc Sci Med 2006; 62: 1768-84.

25 Huang CQ, Dong BR, Lu ZC, Yue JR, Liu QX. Chronic diseases and risk for depression in old age: a meta-analysis of published literature. Ageing Res Rev 2010; 9: 131-41.

क EXTRA 